\title{
Mangiferin Alleviates Renal Interstitial Fibrosis in Streptozotocin- Induced Diabetic Mice through Regulating the PTEN/PI3K/Akt Signaling Pathway
}

\author{
Yanyan Song, ${ }^{1}$ Wei Liu, ${ }^{2}$ Ke Tang, ${ }^{3}$ Junting Zang, ${ }^{4}$ Dong Li $\mathbb{D},{ }^{5}$ and Hang Gao $\mathbb{D}^{4}$ \\ ${ }^{1}$ Department of Nephrology, The Second Hospital of Jilin University, No. 218 Ziqiang Street, Changchun 130021, China \\ ${ }^{2}$ Department of Spinal Surgery, The First Hospital of Jilin University, No. 71 Xinmin Street, Changchun, Jilin 130021, China \\ ${ }^{3}$ Department of Electrical Diagnosis, The Second Hospital of Jilin University, No. 218 Ziqiang Street, Changchun 130021, China \\ ${ }^{4}$ Department of Bone and Joint Surgery, The First Hospital of Jilin University, No. 71 Xinmin Street, Changchun 130021, China \\ ${ }^{5}$ Department of Immunology, College of Basic Medical Sciences, Jilin University, No. 126 Xinmin Avenue, Changchun, \\ Jilin Province 130021, China
}

Correspondence should be addressed to Dong Li; lidong1@jlu.edu.cn and Hang Gao; 13500805575@163.com

Received 8 October 2019; Revised 12 December 2019; Accepted 31 December 2019; Published 1 February 2020

Academic Editor: Akira Sugawara

Copyright (c) 2020 Yanyan Song et al. This is an open access article distributed under the Creative Commons Attribution License, which permits unrestricted use, distribution, and reproduction in any medium, provided the original work is properly cited.

\begin{abstract}
Renal interstitial fibrosis is considered to be the typical manifestation of diabetic nephropathy (DN). Mangiferin has shown positive effect on the prevention or treatment of diabetes and its complications. The aim of this study was to explore the inhibitive effect and mechanism of mangiferin on renal interstitial fibrosis in diabetic mice. Streptozotocin- (STZ-) induced diabetic mice were treated with mangiferin $(15,30$, and $60 \mathrm{mg} / \mathrm{kg} / \mathrm{d})$ for 4 weeks. The morphology of kidneys was observed by Masson's trichrome staining, and the biochemical parameters (fasting blood glucose (FBG), triglyceride (TG), total cholesterol (TC), blood urea nitrogen (BUN), serum creatinine (SCr), and urine protein) were determined by kits. In addition, the levels of inflammatory cytokines (tumor necrosis factor- $\alpha$ (TNF- $\alpha$ ), interleukin- (IL-) 6, and IL-1 $\beta$ ), antioxidant enzymes (SOD, CAT, and GSH-Px), MDA, and ROS were assessed. Furthermore, the expressions of fibronectin (FN), collagen I (Col I), and $\alpha$-SMA were measured by immunohistochemistry. Regulations of TGF- $\beta 1$ and the PTEN/PI3K/Akt pathway were detected by Western blotting. Treatment with mangiferin significantly ameliorated renal dysfunction in diabetic mice, as evidenced by the increase in body weight and decreases in FBG, TG, TC, BUN, SCr, urine protein, and the kidney to body weight ratio (KW/BW). Furthermore, mangiferin treatment prevented renal interstitial fibrosis evidenced by decreases in the positive expression of FN, Col I, and $\alpha$-SMA, in comparison with morphological changes in the renal tissue. Meanwhile, mangiferin increased antioxidant enzymes, reduced the TNF- $\alpha$, IL- 6 , and IL- $1 \beta$, as well as MDA and ROS. Additionally, mangiferin administration also downregulated TGF- $\beta 1$, upregulated PTEN, and decreased the phosphorylation of both PI3K and Akt. These findings demonstrate that mangiferin may reduce inflammation and oxidative stress in DN, thereby inhibiting the renal interstitial fibrosis by reducing the TGF- $\beta 1$-mediated elevation of Col I, FN, and $\alpha$-SMA through the PTEN/PI3K/Akt pathway.
\end{abstract}

\section{Introduction}

Diabetes is a kind of metabolic disease characterized by chronic elevated blood glucose levels. Hyperglycemia is caused by the impaired production of insulin and resistance of insulin [1]. Long-term chronic hyperglycemia induces disorders of fat and protein metabolism, which then causes a series of complications in retinal, kidney, and nerve systems
[2-4]. Diabetic nephropathy (DN) is the most serious complication of diabetes. It is characterized by the loss of renal cells and their replacement by extracellular matrix (ECM), eventually leading to glomerulosclerosis and tubulointerstitial fibrosis [5].

Oxidative stress and chronic inflammation play crucial roles in the development of DN [6, 7]. Hyperglycemia increases the generation of reactive oxygen species (ROS), 
which activate signal transduction and induce the increases of fibrotic factors, such as fibronectin (FN), $\alpha$-smooth muscle actin ( $\alpha$-SMA), and collagen I $[3,8]$. Furthermore, ROS activate the expression of transforming growth factor- (TGF-) $\beta 1$, which contributes to the accumulation of ECM as a key characteristic in renal fibrosis [3,9]. Scientific evidence also suggests the inflammatory factors, tumor necrosis factor- $\alpha$ (TNF- $\alpha$ ) and interleukin-6 (IL-6), are well known to be associated with the development of renal disease in diabetes [10]. In addition, the phosphoinositide 3-kinase (PI3K)/protein kinase $\mathrm{B}$ (Akt) signaling pathway has been demonstrated to play a critical role in proliferation, progression of cell cycle, and cell viability in diabetes [11]. Phosphatase and tensin homologue deleted on chromosome 10 (PTEN) is a negative regulator of the PI3K signaling; it can inhibit the activation of Akt. A significant decrease in the PI3K and Akt was observed in streptozotocin- (STZ-) induced DN of rats [12]. Therefore, antioxidative stress and anti-inflammation are essential approaches for the prevention and treatment of renal fibrosis in DN. It is necessary and urgent to find natural, effective, and safe drugs to treat $\mathrm{DN}$.

Mangiferin (2- $\beta$-D-glucopyranosyl-1,3,6,7-tetrahydroxy9H-xanthen-9-one) is a major active ingredient in the rhizomes of Anemarrhena asphodeloides Bunge, a well-known traditional Chinese medicine [13]. Mangiferin possesses several beneficial biological activities such as antioxidant, antimicrobial, antidiabetic, antiallergic, anticancer, hypocholesterolemic, and immunomodulatory $[14,15]$. The reports suggest that mangiferin has a positive effect on the prevention or treatment of diabetes and its complications. Although the beneficial effects of mangiferin on DN have also been confirmed in previous studies, reports regarding the mechanisms of mangiferin on renal interstitial fibrosis in DN are limited.

In this study, STZ-induced diabetic mice were used as models to study the protective effect of mangiferin on diabetic renal interstitial fibrosis injury and to explore the mechanism of the PTEN/PI3K/Akt signaling pathway in mangiferin inhibiting renal interstitial fibrosis in DN, which might be able to provide more theoretical evidence for clinical application of traditional Chinese medicine on treatment of diabetes.

\section{Materials and Methods}

2.1. Mice. A total of $70 \mathrm{C} 57 \mathrm{BL} / 6$ male mice (7 weeks old) weighing $21 \mathrm{~g} \pm 2 \mathrm{~g}$ were obtained from the Experimental Animal Center at Jilin University (Jilin, China). The experiments had been approved by the ethics committee of the Second Hospital of Jilin University. All animal experiments were performed in accordance with the National Guidelines for Experimental Animal Welfare and with approval of the Animal Welfare and Research Ethics Committee at Jilin University (Changchun, China). The mice were housed in the SPF condition with constant 22 to $25^{\circ} \mathrm{C}$ room temperature, 45-55\% humidity, a 12-hour light-dark cycle, and accessible clean food and water ad libitum.

The diabetic mice models were performed as described with some modifications [3]. After acclimatization for one week, mice were divided into two groups; the mice in the control group (Con, $N=10$ ) were treated with citric acid buffer, whereas the model mice $(N=60)$ were given injection with multiple low-dose STZ $(50 \mathrm{mg} / \mathrm{kg}$, Sigma Aldrich, St. Louis, MO, USA). Injections were repeated in 5 consecutive days. STZ was dissolved in $0.1 \mathrm{~mol} / \mathrm{L}$ ice-cold citric acid buffer ( $\mathrm{pH}$ 4.5), and the injection was completed within $30 \mathrm{~min}$. Mice with fasting blood glucose (FBG) higher than $13.9 \mathrm{mmol} / \mathrm{L}(250 \mathrm{mg} / \mathrm{dL})$ after $72 \mathrm{~h}$ were established as successful diabetes model mice. Mangiferin (>97\% purity, China Food and Drug Regulatory Research Institute, Beijing, China) was suspended in distilled water and was given to the diabetic mouse by oral gavage once daily. Bisperoxovanadium (BpV, HOpic) (Selleck Chemicals, USA) is a highly potent inhibitor of PTEN with an $\mathrm{IC}_{50}$ of $14 \mathrm{nM}$. Diabetic mice were divided into 6 groups randomly $(N=10)$ : model group (Mod), mangiferin in low dose group (Mang-L, $15 \mathrm{mg} / \mathrm{kg} / \mathrm{d}$ ), mangiferin in middle dose group (Mang-M, $30 \mathrm{mg} / \mathrm{kg} / \mathrm{d}$ ), mangiferin in high dose group (Mang- $\mathrm{H}$, $60 \mathrm{mg} / \mathrm{kg} / \mathrm{d}$ ), PTEN inhibitor group (BpV, diabetic mice were injected with PTEN inhibitor and given normal saline), and PTEN inhibitor+Mangiferin group $(\mathrm{BpV}+\mathrm{Mang}-\mathrm{H}$, diabetic mice were injected with PTEN inhibitor and given mangiferin $60 \mathrm{mg} / \mathrm{kg} / \mathrm{d}$ ).

2.2. Assessment of Biochemical Parameters. The body weights (BW) of the mice were weighted before sacrificed. The mice were sacrificed by anesthetizing with ketamine $(30 \mathrm{mg} / \mathrm{kg})$ and thiobutabarbital $(50 \mathrm{mg} / \mathrm{kg}$ ) after experimental 4 weeks. The blood was collected in test tubes with heparin solution via the caudal vena cava, followed by serum separation. The urine was collected from the bladder to measure the urine protein. Fasting blood glucose (FBG), triglyceride (TG), total cholesterol (TC), blood urea nitrogen (BUN), serum creatinine ( $\mathrm{SCr}$ ), and urine protein in the urine and serum were measured according to the manufacturer's protocol for each kit (Jiancheng Bioengineering Institute, Nanjing, China). The kidneys were collected and weighted to calculate the kidney to body weight ratio $(\mathrm{KW} / \mathrm{BW})$. The samples were stored at $-80^{\circ} \mathrm{C}$ for further analysis.

2.3. Histological Analysis. Masson's trichrome staining was performed as described before [13] with some modifications. The kidney tissue was fixed in $10 \%$ formalin, and routinely paraffin-embedded, $4 \mu \mathrm{m}$ thick sections of the sample were dewaxed, hydrated, and then stained with Masson's trichrome, cleared in xylene, and mounted with neutral balsam. The stained sections were examined under a light microscope (Olympus BX-50, Japan) at 400x magnification by two blinded pathologists. Three points in the central sections of each lesion were analyzed, expressed as the approximate proportion of stained collagen fibers to the total stained part. Image Pro Plus 6.0 was used to analyze the optical density (OD) of image results.

2.4. Measurement of ROS. The kidney tissue was homogenized with saline $(1: 9 \mathrm{w} / \mathrm{v})$ at $4^{\circ} \mathrm{C}$. The homogenates were centrifuged at $11000 \mathrm{~g}$ for $15 \mathrm{~min}$ at room temperature. ROS production was measured by using a 2,7-dichlorofluorescein diacetate (DCFH-DA) kit (Beyotime Biotech Inc., Shanghai, 
China). The fluorescence was collected by a microplate reader (BioTek Instruments, USA) equipped with a fluorescence detector (the excitation wavelength was $488 \mathrm{~nm}$, and emission wavelength was $510 \mathrm{~nm}$ ).

2.5. Measurement of MDA and Antioxidant Enzymes. The kidney tissue was homogenized, and protein concentration was measured using a BCA protein assay kit (Beyotime Biotech Inc., Shanghai, China). Malondialdehyde (MDA) content, levels of superoxide dismutase (SOD), catalase (CAT), and glutathione peroxidase (GSH-Px) were measured using commercial kits (Beyotime Biotech Inc., Shanghai, China) according to the manufacturer's instructions.

2.6. Analysis of Inflammatory Factors in Kidney Tissues. The interleukin-6 (IL-6, No. PI330), interleukin-1 $\beta$ (IL-1 $\beta$, No. PI305), and tumor necrosis factor- $\alpha$ (TNF- $\alpha$, No. PT518) concentrations in the renal homogenates were measured using commercial ELISA kits (Beyotime Biotech Inc., Shanghai, China) according to the manufacturer's instructions.

2.7. Immunofluorescence (IF) Staining. Immunofluorescence staining was performed as described before [16] with some modification, to detect the expression levels and differences of fibronectin (FN), collagen I ( $\mathrm{Col} \mathrm{I}$ ), and $\alpha$-smooth muscle actin ( $\alpha$-SMA) in the kidneys of five groups (Mod group, Mang- $\mathrm{H}$ group, $\mathrm{BpV}$ group, and $\mathrm{BpV}+\mathrm{Mang}-\mathrm{H}$ group). The sections of the paraffin-embedded sample were made and blocked with serum-free protein (Dako, Victoria, Australia) and permeabilized for $30 \mathrm{~min}$. Sections were incubated with primary antibodies FN, Col I, $\alpha$-SMA (Wanleibio, Shijiazhuang, China; $1: 100$ dilution) overnight at $4^{\circ} \mathrm{C}$. Sections were washed with TBS $3 \times 10 \mathrm{~min}$ and incubated with species-specific secondary antibodies: Goat AntiRabbit IgG H\&L (Cy3 $\left.{ }^{\circledR}\right)(1: 1000$ dilution, ab6939, Abcam, USA) at room temperature for $1 \mathrm{~h}$. After washing with TBS, the sections were stained with Glycerol Mounting Medium (Abcam) that contained 4,6-diamidino-2-phenylindole (DAPI) and 1,4-diazobicyclo-2,2,2-octane (DABCO). Labelled tissues were visualized using a Leica DM LB2 microscope. Fluorescence images (400x magnification) were captured using NIS-Elements 4.13 (Nikon, Japan) software. The number of fluorescence-positive cells was counted from five representative high-power fields (400x magnification) per tissue section.

2.8. Western Blot. Total protein concentration of homogenate was measured using a BCA protein assay kit (Beyotime Biotech Inc., Shanghai, China) according to the manufacturer's protocol and was equalized before electrophoresis. Briefly, $40 \mu \mathrm{g}$ of the proteins in the supernatant was separated by $10 \%$ SDS-PAGE and transferred onto PVDF membranes. After blocking at room temperature for $1 \mathrm{~h}$ with $5 \%$ nonfat dry milk, the membranes incubated with antibodies TGF$\beta 1$ (1:500 dilution, Wanleibio, Shijiazhuang, China), PTEN (1:400 dilution, Wanleibio, Shijiazhuang, China), PI3K p85 (1:1000 dilution, Cell Signaling Technology, USA), p-PI3K p85 (Tyr458)/p55 (Tyr199) (1:1000 dilution, Cell Signaling Technology, USA), and Akt and p-Akt (Ser473) $(1: 1000$ dilution, Cell Signaling Technology, USA) overnight at $4^{\circ} \mathrm{C}$.
After washing with TBST, the membranes were incubated with IgG-HRP (1:5000 dilution, Wanleibio, Shijiazhuang, China) for $1 \mathrm{~h}$ at room temperature. The membranes were developed with enhanced chemiluminescence using ECL reagents (Beyotime Biotech Inc., Shanghai, China) and visualized using a digital imaging system (Bio-Rad Laboratories, Inc., USA). The blots were quantitated by densitometric analysis using NIH ImageJ software. The data were normalized on the basis of GAPDH level.

2.9. Statistical Analysis. Data were expressed as mean \pm standard error of mean (SEM). Differences between groups were assessed with one-way analysis of variance (ANOVA), followed by Tukey's test using the SPSS 21.0 statistical package (IBM Corp., Armonk, NY). Differences were considered statistically significant at $P<0.05$.

\section{Results}

3.1. Mangiferin Reduces FBG and Elevates Body Weight of STZ-Induced Diabetic Mice. As shown in Figures 1(a) and 1(b), FBG was found to be significantly elevated in STZinduced diabetic mice as compared to normal mice $(P<0.05)$. At weeks 1, 2, 3, and 4, treatment with mangiferin $(15,30$, and $60 \mathrm{mg} / \mathrm{kg} / \mathrm{d}$ ) resulted in a concentration-dependent reduction in FBG as compared to model mice $(P<0.05)$, but not completely reduced to the level of normal mice (Figures 1(a) and 1(b)). The body weights of model mice were significantly decreased compared with the normal mice. After treated with mangiferin, the body weights were increased in a dose-dependent manner compared with the model mice $(P<0.05$, Figure $1(\mathrm{c}))$. These results indicate that mangiferin exhibits antidiabetic effect on STZ-induced diabetic mice.

3.2. Mangiferin Alleviates Kidney Dysfunction and Lipid Metabolism of Diabetic Mice. Specific markers related to kidney dysfunction such as BUN, $\mathrm{SCr}$, and urine protein, as well as the kidney to weight ratio (KW/BW), were shown in Figure 2. The significant elevations of BUN, SCr, and urine protein were observed in model mice $(P<0.05$, Figures $2(\mathrm{a})-2(\mathrm{c}))$. However, mangiferin treatment efficiently reduced the elevations of these biochemical parameters. In addition, the $\mathrm{KW} / \mathrm{BW}$ of diabetic mice also reduced by mangiferin compared with the model mice $(P<0.05$, Figure $2(\mathrm{~d}))$. Furthermore, treatment with mangiferin significantly reduced the levels of TG and TC in dose-dependent manners, which were elevated in the model mice $(P<0.05$, Figures $2(\mathrm{e})$ and $2(\mathrm{f}))$. These results indicate that mangiferin exhibits protective effects on renal injuries and lipid metabolism disorders in STZ-induced diabetic mice.

3.3. Mangiferin Protects against the Renal Injury of STZInduced Diabetic Mice. Masson's trichrome staining was used to observe the effects of mangiferin on the renal fibrosis of diabetic mice. The collagen fiber was stained blue, and the muscle fiber cytoplasm was stained red. These changes in the glomerulus of the control and experimental groups are shown in Figure 3. A little renal collagen fiber deposition was observed in the untreated mice (Con group). But in the 


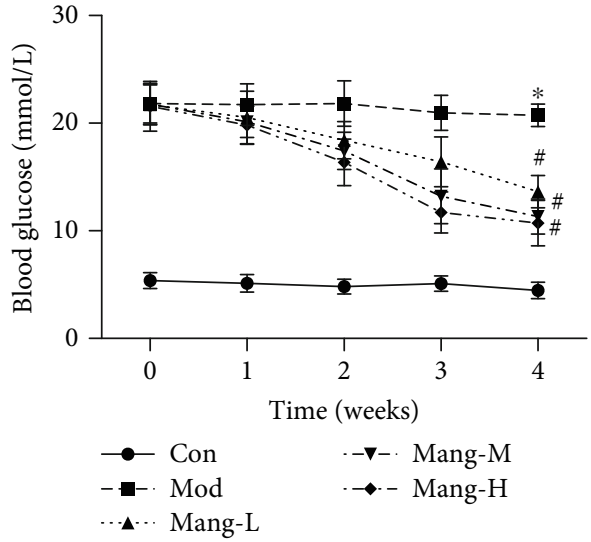

(a)

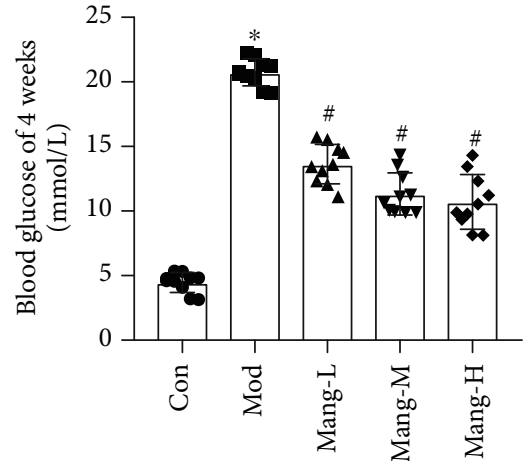

(b)

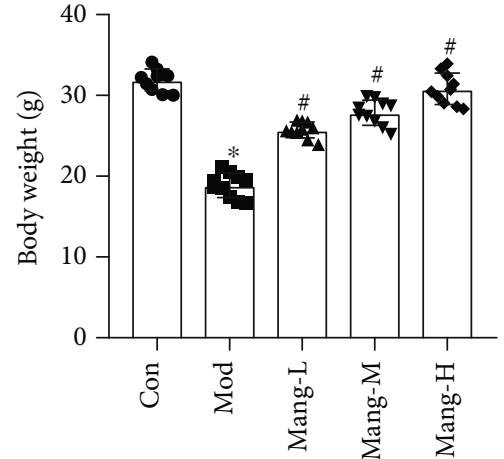

(c)

FIGURE 1: Effects of mangiferin on fasting blood glucose (FBG) and body weight of diabetic mice. (a) FBG of weeks 1, 2, 3, and 4. (b) FBG of week 4. (c) Body weight. Data are expressed as the mean \pm S.D., $N=10,{ }^{*} P<0.05$ versus the Con group, ${ }^{\#} P<0.05$ versus the Mod group.

Mod group, the basement membrane of tubules was thickened, and renal interstitial fibrosis was observed. A significant increase of collagen volume was observed in the tubulointerstitium of mice treated with STZ alone (Mod group, $P<0.05$, Figure $3(\mathrm{~b})$ ). These changes of mesenchymal collagen fiber were effectively alleviated with mangiferin treatment (Mang-L, Mang-M, and Mang-H) with dosedependent manners $(P<0.05$, Figure $3(\mathrm{~b}))$. Especially in high-dose-treated mice, the visible collagen fibers were quite near to that of the control group. These results further show that mangiferin exhibit renal protective effect on DN.

\subsection{Mangiferin Reduces the Elevated Levels of Inflammatory} Factors in Diabetic Mice. The TNF- $\alpha$ level increased in the model group, while the administration of mangiferin was found to decrease the level of TNF- $\alpha$ in kidney in a dosedependent manner $(P<0.05$, Figure $4(\mathrm{a}))$. In addition, treatment with mangiferin also decreased the levels of IL- $1 \beta$ and IL-6 in dose-dependent manners compared to the model group, which were significantly increased in diabetic mice in comparison with the normal mice $(P<0.05$, Figures $4(\mathrm{~b})$ and $4(\mathrm{c}))$. These results suggest that the protective effect of mangiferin on diabetic renal injury is partly due to its antiinflammatory effect.

3.5. Mangiferin Plays an Antioxidant Role in STZ-Induced Diabetic Mice. As shown in Figure 5, the antioxidant enzyme activities (SOD, CAT, and GSH-Px) were significantly decreased in model mice, along with the significant elevation of MDA and ROS levels $(P<0.05)$, while treatment with mangiferin increased the activities of SOD, CAT, and GSH$P x$ in a dose-dependent manner $(P<0.05$, Figures 5(a)5(c)). Also, the decreasing MDA and ROS levels were found with administration of mangiferin in a dose-dependent manner $(P<0.05$, Figures $5(\mathrm{~d})$ and $5(\mathrm{e}))$. These results suggest that the protective effect of mangiferin on diabetic renal injury partly results from its antioxidative stress effect.

3.6. Mangiferin Alleviates the Renal Fibrosis of STZ-Induced Diabetic Mice. To further investigate the protective effect of mangiferin on the renal fibrosis of diabetic mice, the expressions levels and differences of ECM-related factors, such as fibronectin (FN), collagen I ( $\mathrm{Col} \mathrm{I}$ ), and $\alpha$-SMA in the renal cortex, were observed in Figure 6. Compared with the normal group, the expressions of FN, Col I, and $\alpha$-SMA were significantly increased in kidneys of diabetic mice $(P<0.05)$. Nevertheless, treatment with high-dose mangiferin effectively reversed the elevation of FN, Col I, and $\alpha$-SMA $(P<0.05)$. Additionally, with the use of the PTEN inhibitor (BpV), it was found that the expressions of FN, Col I, and $\alpha$-SMA were significantly higher than those of model mice $(P<0.05)$. However, mangiferin treatment also exhibited significant decreases of FN, Col I, and $\alpha$-SMA levels in the BpVtreated diabetic mice $(P<0.05)$ but did not surpass the mangiferin treatment group without PTEN inhibitor. These results suggest that the protective effect of mangiferin on renal interstitial fibrosis is related to its inhibitory effect on generation and accumulation of ECM factors.

3.7. Mangiferin Regulated TGF- $\beta 1$ and the PTEN/PI3K/Akt Signaling Pathway. In order to be more definitive, the related signaling pathways that regulate ECM were evaluated. Figures 7(a) and 7(b) show the inhibitive effect of mangiferin on the upstream TGF- $\beta 1$ signaling. Compared with the normal group, the expression of TGF- $\beta 1$ was significantly increased in kidneys of diabetic mice $(P<0.05)$, while treatment with high-dose mangiferin effectively reversed the elevation of TGF- $\beta 1(P<0.05)$, which was consistent with the expressions of FN, Col I, and $\alpha$-SMA close to the normal level. Additionally, with the intervention of $\mathrm{BpV}$, expression of TGF- $\beta 1$ was significantly higher than that of model mice $(P<0.05)$. Nevertheless, compared with the $\mathrm{BpV}$ group, mangiferin treatment significantly decreased the TGF- $\beta 1$ expression in the $\mathrm{BpV}$-treated diabetic mice $(P<0.05)$. These results suggest that the protective mechanism of mangiferin on renal interstitial fibrosis in part associates with the inhibitory of TGF- $\beta 1$-mediated ECM elevation.

The inhibitive effect of mangiferin on the PTEN/PI3K/Akt signaling pathway was shown in Figures 7 (a) and $7(\mathrm{c})-7(\mathrm{e})$. In the model group, the protein expressions of $\mathrm{p}$ - 


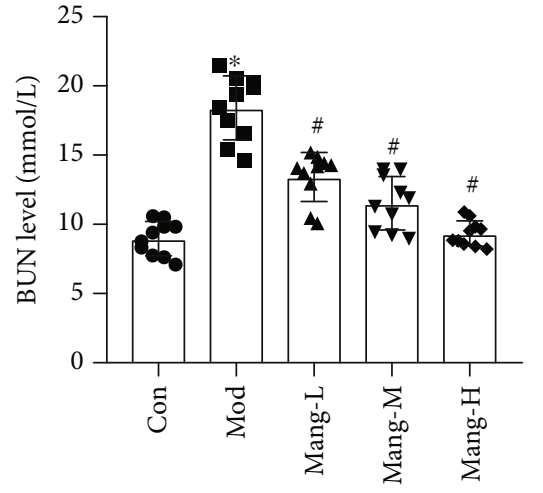

(a)

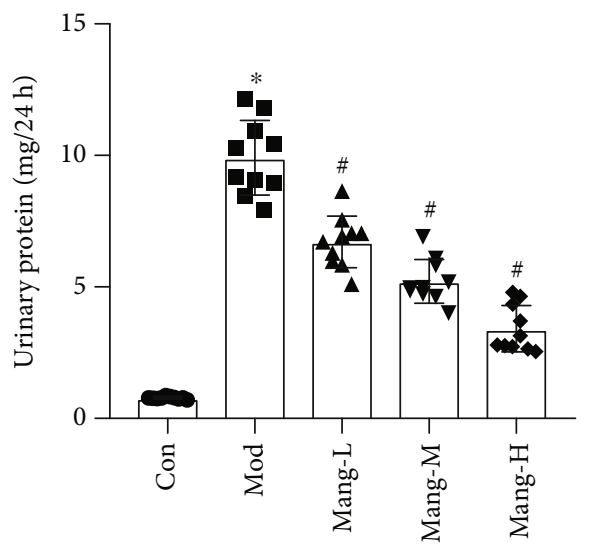

(c)

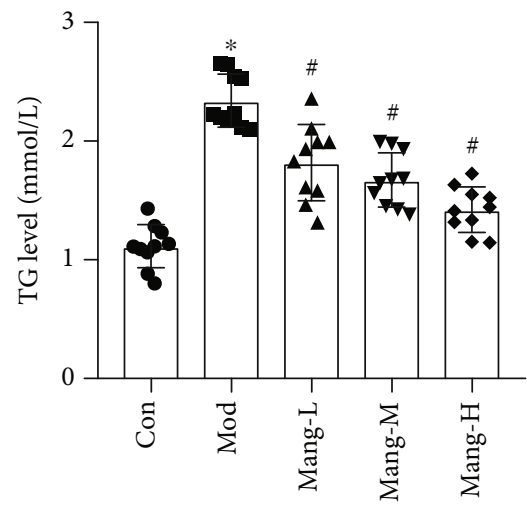

(e)

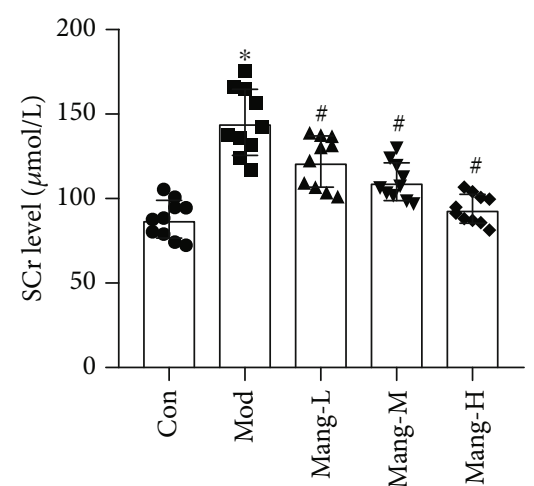

(b)

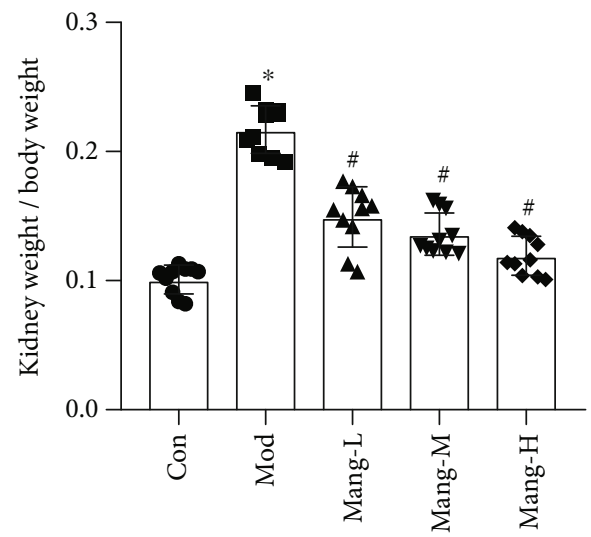

(d)

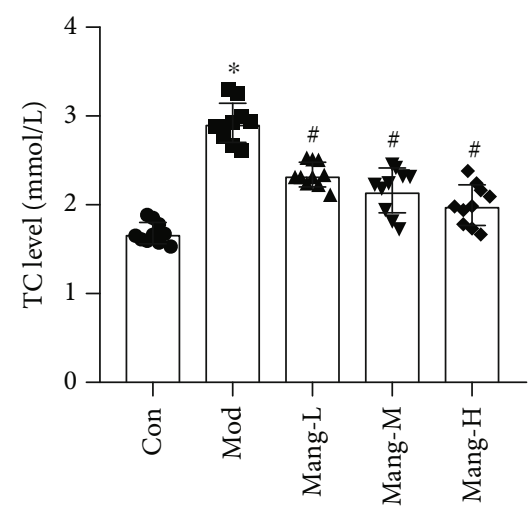

(f)

Figure 2: Effects of mangiferin on biochemical parameters of kidney dysfunction and lipid metabolism in diabetic mice. (a) Blood urea nitrogen (BUN) level. (b) Serum creatinine (SCr) level. (c) Urine protein level. (d) Kidney to body weight ratio (KW/BW). (e) Triglyceride (TG) level. (f) Total cholesterol (TC) level. Data are expressed as the mean \pm S.D., $N=10,{ }^{*} P<0.05$ versus the Con group, ${ }^{\sharp} P<0.05$ versus the Mod group.

PI3K/PI3K and p-Akt/Akt were significantly higher than those in the normal group $(P<0.05)$; on the contrary, the expression of PTEN was significantly decreased $(P<0.05)$. With the intervention of $\mathrm{BpV}$, expressions of $\mathrm{p}-\mathrm{PI} 3 \mathrm{~K} / \mathrm{PI} 3 \mathrm{~K}$ and $\mathrm{p}$-Akt/Akt were further increased compared to model mice $(P<0.05)$. However, compared with the $\mathrm{BpV}$ group, mangiferin treatment significantly decreased the p-PI3K/PI3K and p-Akt/Akt expressions and simultaneously increased PTEN proteins in the $\mathrm{BpV}$-treated diabetic mice $(P<0.05)$. These findings demonstrate that mangiferin increases resistance to renal interstitial fibrosis by modulating the PTEN/PI3K/Akt signaling pathway, which can serve not only as a noninvasive biomarker but also as a pathologic mediator and therapeutic target of kidney fibrosis.

\section{Discussion}

Diabetic nephropathy (DN) is one of the most severe diabetic complications. Renal fibrosis, especially glomerulosclerosis and tubulointerstitial fibrosis, is considered to be the final 

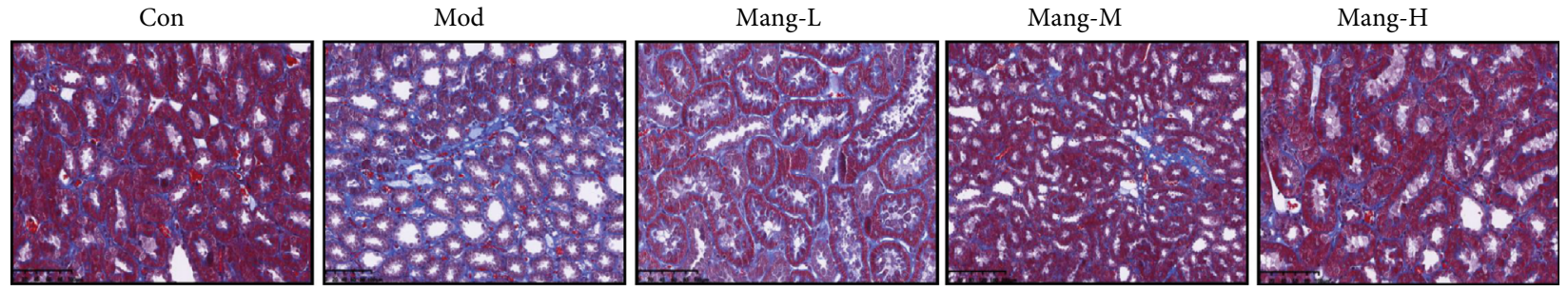

(a)

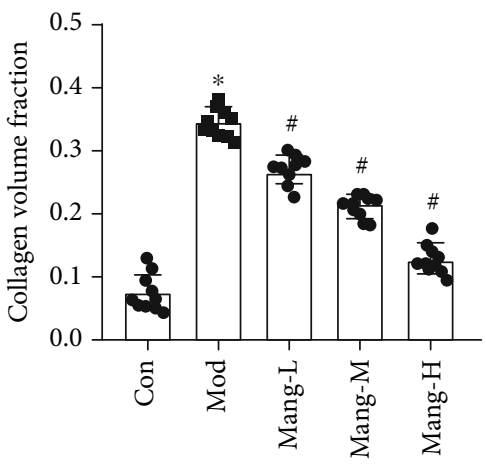

(b)

Figure 3: The histopathology of mangiferin effects on renal injury in STZ-induced diabetic mice. (a) Masson's trichrome staining, $\times 400$ $($ Scale bar $=50 \mu \mathrm{m})$. (b) Semiquantitative analysis of stained positive area in the lesions. Data are expressed as the mean \pm S.D., $N=10$, ${ }^{*} P<0.05$ versus the Con group, ${ }^{\#} P<0.05$ versus the Mod group.

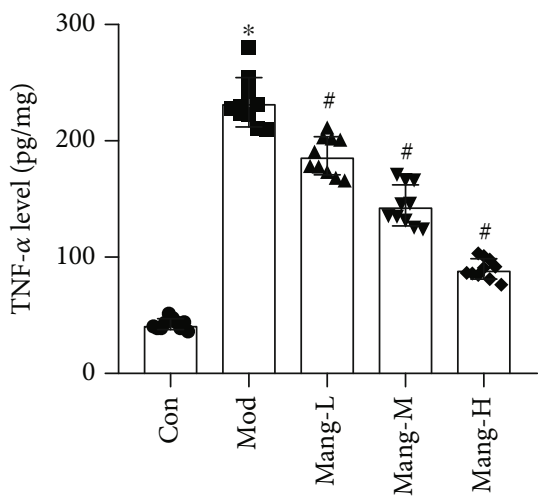

(a)

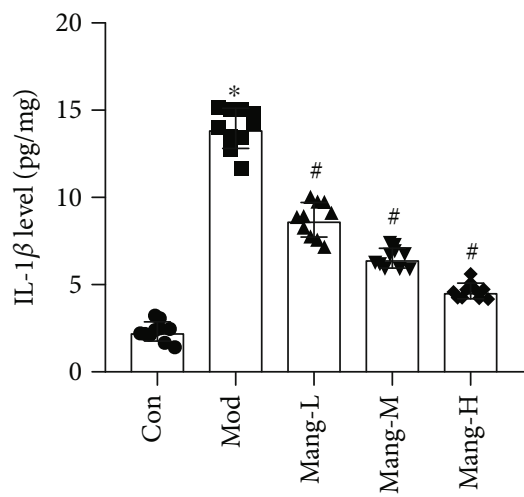

(b)

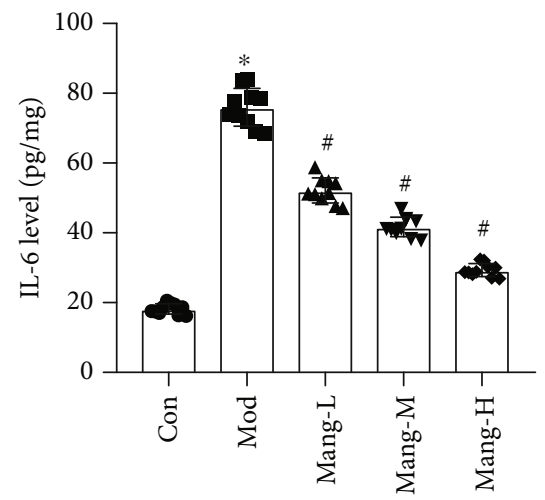

(c)

FIGURE 4: Effects of mangiferin on inflammation in the kidney of STZ-induced diabetic mice. (a) Tumor necrosis factor- $\alpha$ (TNF- $\alpha$ ) level. (b) Interleukin-1 $\beta$ (IL-1 $\beta$ ) level. (c) Interleukin-6 (IL-6) level. Data are expressed as the mean \pm S.D., $N=10,{ }^{*} P<0.05$ versus the Con group, ${ }^{\#} P<0.05$ versus the Mod group.

manifestations of DN [17]. In order to develop effective preventable and therapeutic strategies of $\mathrm{DN}$, it is essential to understand the cellular and molecular mechanisms underlying fibrotic development. Mangiferin is a xanthone present in Anemarrhena asphodeloides Bunge. It exhibits tremendous health-related properties such as antiviral, anticancer, antidiabetic, antioxidative, antiaging, immunomodulatory, hepatoprotective, and analgesic effects. In previous studies, mangiferin exhibited beneficial effects on diabetes and diabetic complications, especially focused on $\mathrm{DN}[13,18,19]$. The current study investigated the effects of mangiferin on the renal fibrosis of diabetic mice and expounded the underlying mechanisms associated with the PTEN/PI3K/Akt signaling pathway. The present study suggested that the potential abilities of mangiferin on $\mathrm{DN}$ in vivo were related to the inhibitory effect of renal interstitial fibrosis.

STZ is usually used to establish the animal diabetic model; one of the characteristics in pathology is inducing diabetic renal changes $[20,21]$. Thus, a continued low dose of STZ-induced diabetic mice was selected as model for investigating the effect of mangiferin on renal injury. Elevated glucose levels successfully induced renal lesions that were similar with human patients of DN, which is characterized by hyperglycemia, hyperlipidemia, oxidative stress, and renal damage [22]. In the present study, STZ-induced diabetic mice showed development of renal injury by histomorphological and biochemical analysis, which was significantly ameliorated by mangiferin treatment. The results demonstrated 


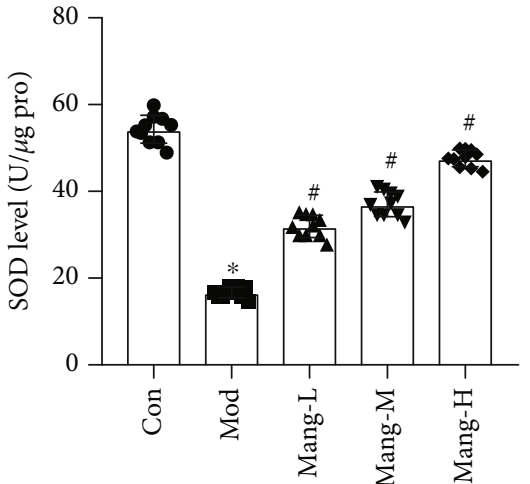

(a)

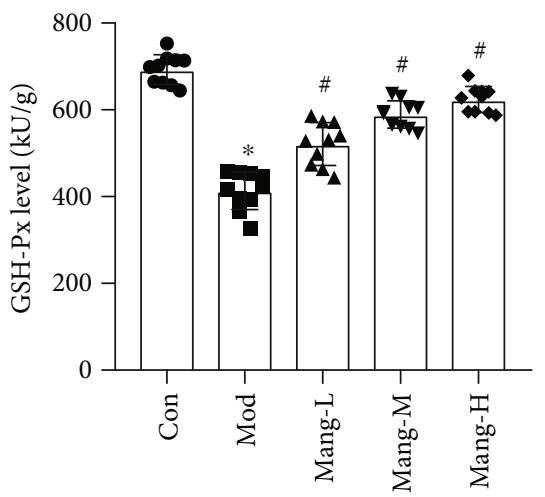

(c)

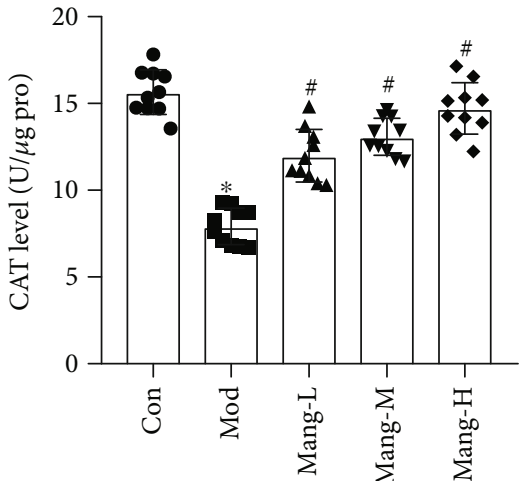

(b)

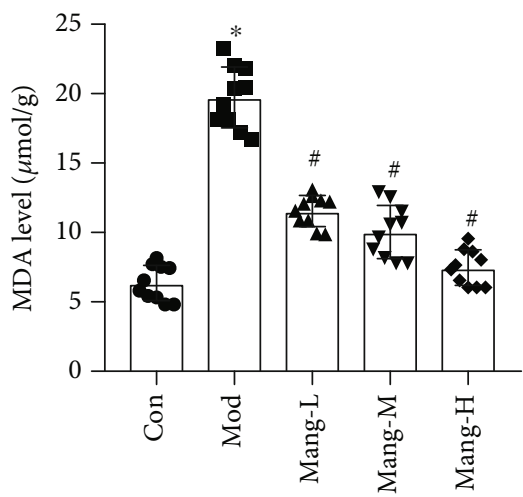

(d)

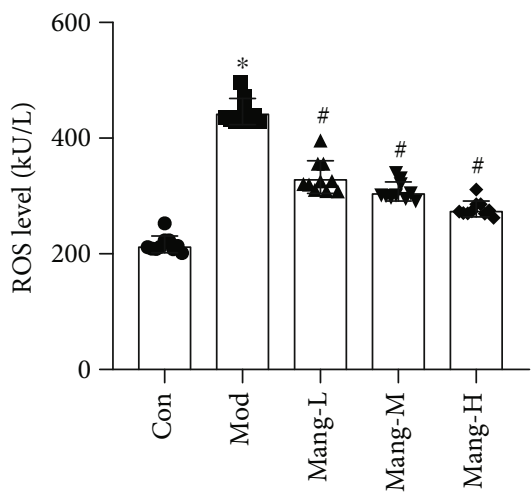

(e)

FIGURE 5: Effects of mangiferin on antioxidant activities in the kidney of STZ-induced diabetic mice. (a) Superoxide dismutase (SOD) level. (b) Catalase (CAT) level. (c) Glutathione peroxidase (GSH-Px) level. (d) Malondialdehyde (MDA) level. (e) Reactive oxygen species (ROS). Data are expressed as the mean \pm S.D., $N=10,{ }^{*} P<0.05$ versus the Con group, ${ }^{\#} P<0.05$ versus the Mod group.

that mangiferin could decrease the FBG levels and increase $\mathrm{KW} / \mathrm{BW}$ ratios of diabetic mice. A previous study also revealed that mangiferin reduced the plasma glucose level and restored kidney to body weight ratio [7]. The biochemical levels including SCr and BUN are critical indicators of DN which gradually increased in diabetic patients and in turn further accelerated the development of DN [23, 24]. In this study, mangiferin significantly decreased SCr, BUN, and urine protein in STZ-induced diabetic mice, which meant the mangiferin could partially mitigate the progression of DN. These further confirmed the beneficial effects of mangiferin on diabetes, focused on glycemic control and renal protection. The improved effect of mangiferin on renal fibrosis may also be related to regulating FBG level, which also contributes to its effects that we cannot ignore. However, in this study, although mangiferin can reduce FBG, it did not reduce to the normal level of control. From this, it can be speculated that the improvement of mangiferin on renal fibrosis is not completely determined by the inhibitory effect of glycemia.

Abnormal lipid metabolism often occurs in the pathogenesis of DN [25]. As the primary lipid metabolic factors, TC and TG, are typically increased in diabetic patients [26]. In the study, TC and TG levels were significantly decreased after mangiferin treatment in the STZ-induced diabetic mice, indicating that mangiferin can benefit diabetic mice via the 


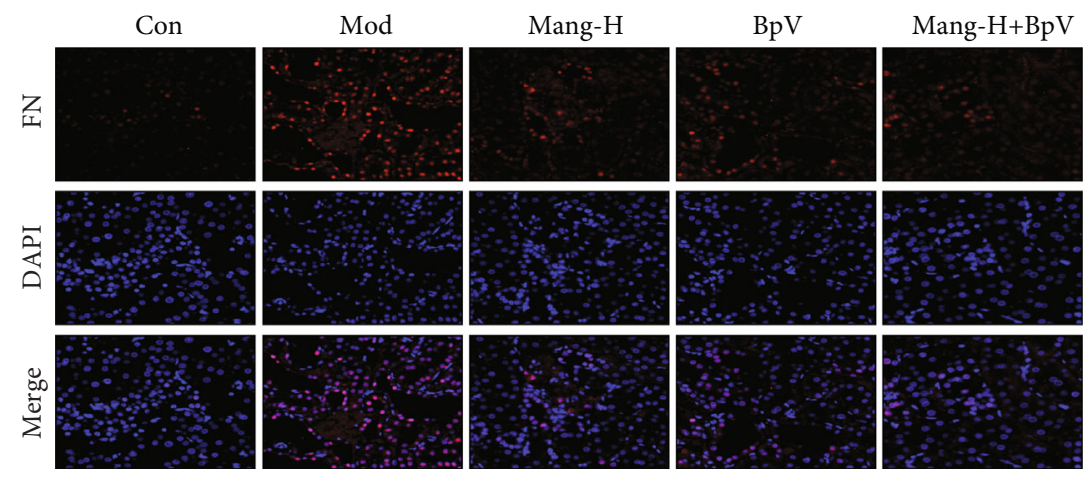

(a)

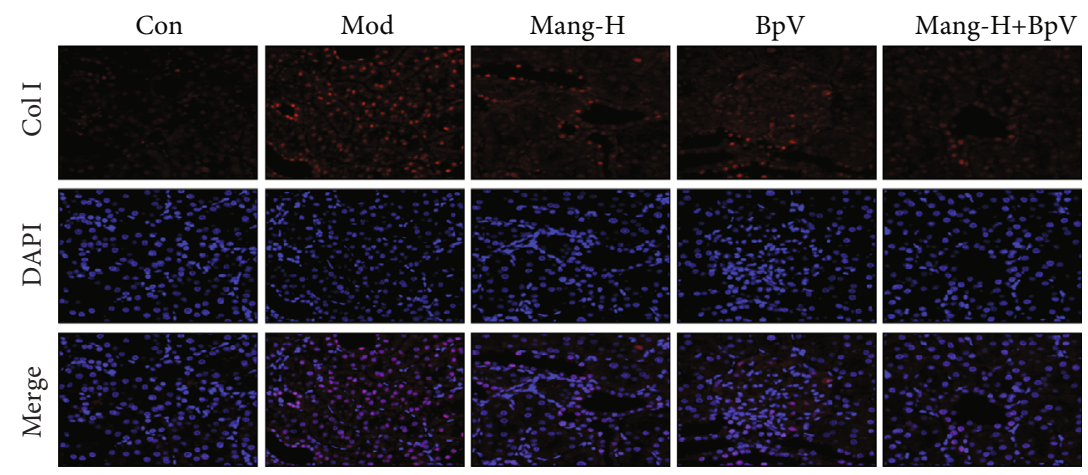

(b)

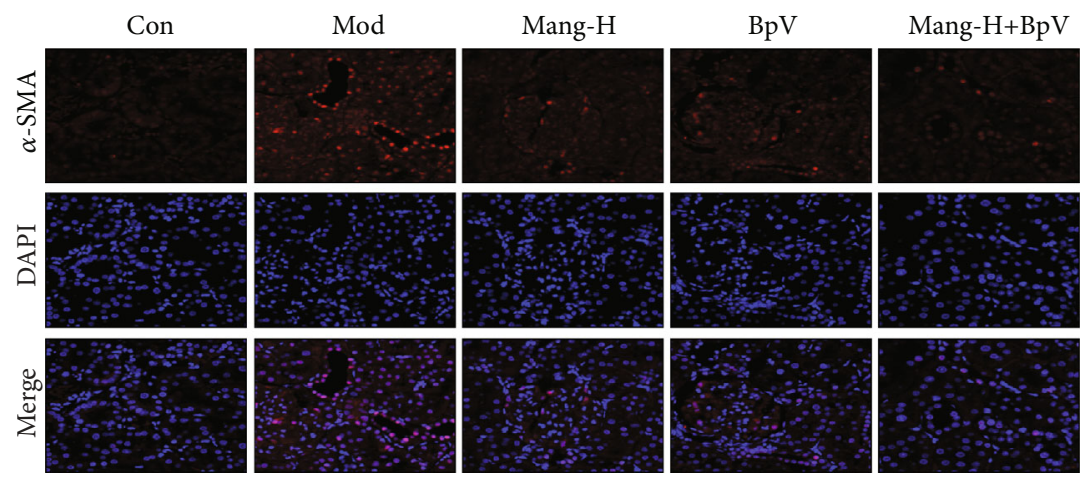

(c)

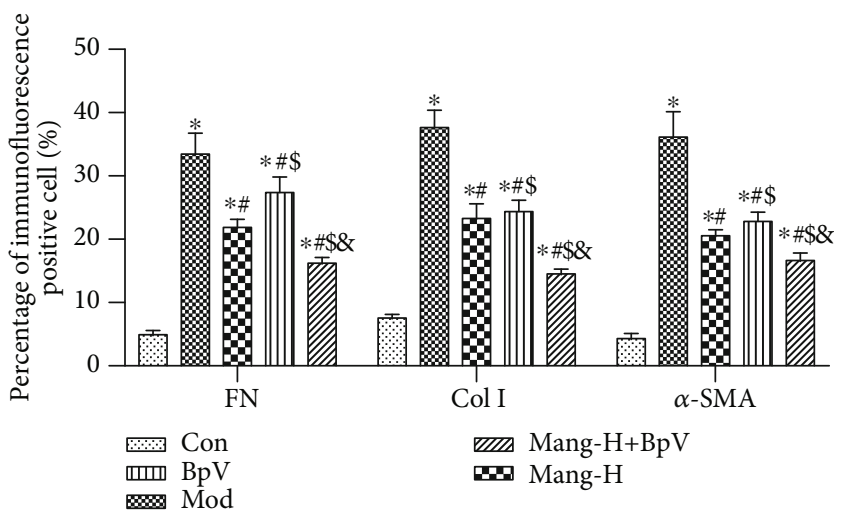

(d)

FIGURE 6: Immunofluorescence analysis for the effect of mangiferin on expressions of fibronectin (FN), collagen I (Col I), and $\alpha$-smooth muscle actin ( $\alpha$-SMA) in diabetic mice. (a) Immunofluorescence of fibronectin (FN). (b) Immunofluorescence of collagen I (Col I). (c) Immunofluorescence of $\alpha$-smooth muscle actin ( $\alpha$-SMA). (d) Quantitative results. ${ }^{*} P<0.05$ versus the Con group, ${ }^{\#} P<0.05$ versus the Mod group, ${ }^{\$} P<0.05$ versus the Mang-H group, ${ }^{\circledR} P<0.05$ versus the $\mathrm{BpV}$ group. ns: no significance. 


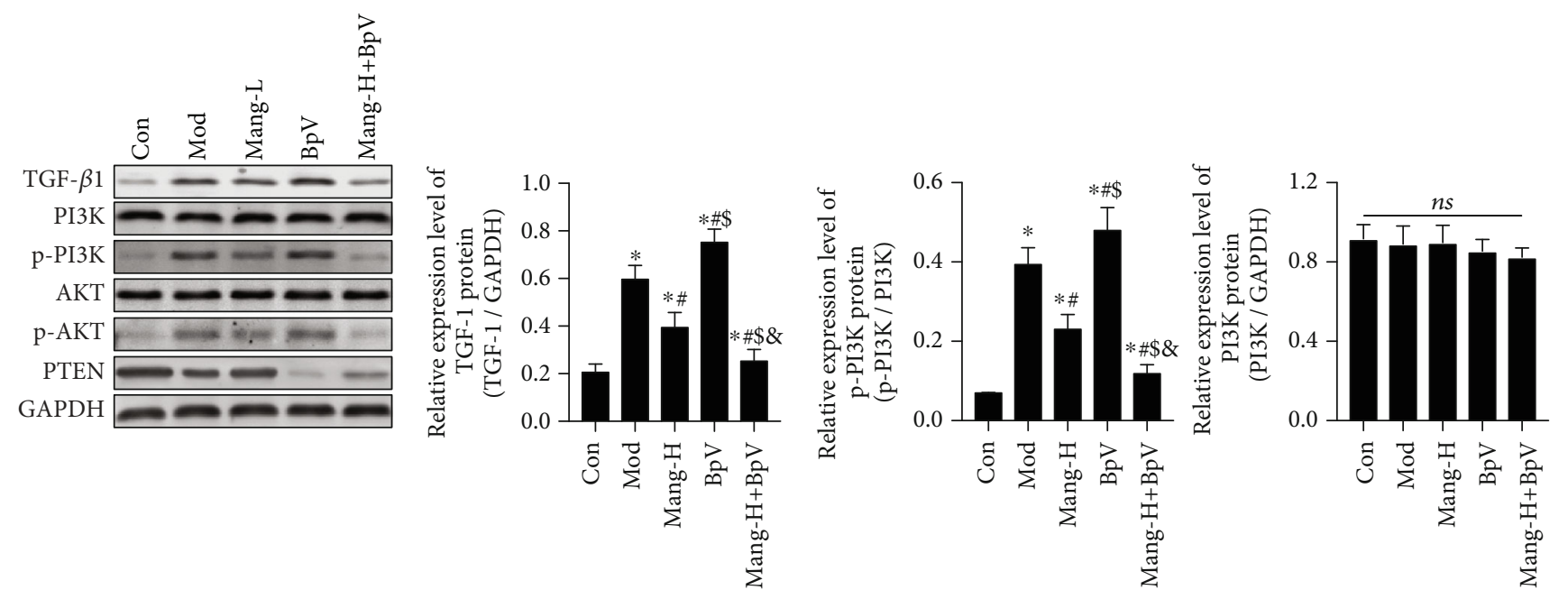

(a) (b)

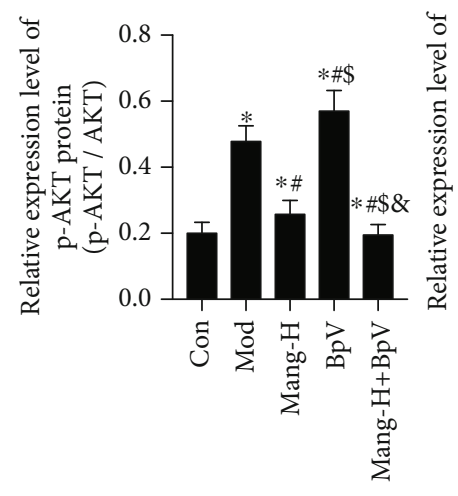

(d)

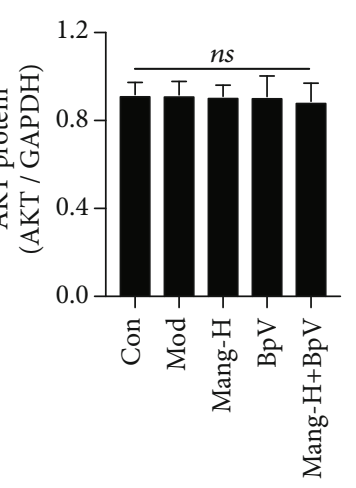

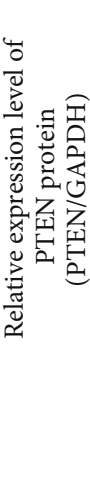

(c)

FIGURE 7: Effects of mangiferin on TGF- $\beta 1$ and the PTEN/PI3K/Akt signaling pathway. (a) Western blot band. (b) The protein expressions of TGF- $\beta 1$. (c) The protein expressions of p-PI3K p85 (Tyr458)/p55 (Tyr199) and PI3K p85. (d) The protein expressions of p-Akt (Ser473) and Akt. (e) The protein expressions of PTEN. Data are expressed as the mean \pm S.D., $N=4,{ }^{*} P<0.05$ versus the Con group, ${ }^{\#} P<0.05$ versus the Mod group, ${ }^{\$} P<0.05$ versus the Mang-H group, ${ }^{\circledR} P<0.05$ versus the $\mathrm{BpV}$ group. ns: no significance.

regulation of abnormal lipid metabolism and dyslipidemia. These results are consistent with the relevant research about inhibitory effect of timosaponin B-II on elevated blood lipids in alloxan-induced mice [27]. Similarly, the flavonoids of A. asphodeloides Bunge were reported to reduce FG and TG in type 2 DM rats [28]. The antidiabetic drug metformin improves lipid metabolism and ameliorates lipid peroxidation via enhancing insulin sensitivity [3]. Thus, mangiferin might undergo a similar mechanism to regulate $\mathrm{DN}$-induced dysfunction of lipid metabolism.

In the progress of $\mathrm{DN}$ in diabetic patients, the renal hypertrophy, nodular sclerosis, thickening of the GBM, and no proliferation of mesangial matrix are pivotal pathological characteristics [27]. In the current study, STZ-induced diabetic mice showed that remarkable fibrosis of tubulointerstitium accompanied these features, which were alleviated by mangiferin. The result demonstrated that mangiferin could markedly improve the renal injury to inhibit DN in mice. These are consistent with the previous reports [7, 17]. Relevant study also indicated that TB-II treatment ameliorated the suppression of nodular sclerosis and alleviated glomerular injuries [27]. A study demonstrated that mangiferin shows beneficial preventable and remedial effects on renal fibrosis [13]. Further, the potential mechanism was revealed.

A previous study has revealed that targeting oxidative stress and inflammation could improve therapeutic options for DN [29]. A hypothesis has been proposed that overproduction of ROS induced by high blood glucose may result in various pathogenic pathways in DN [30]. Various studies showed that mangiferin can protect kidney based on its effectively endogenous antioxidant system regulating and strong free radical (ROS) scavenging activity in diabetes [7, 17, 29]. In this study, the activities of SOD, CAT, and GSH-Px increased, accompanied by decreases of MDA and ROS levels after mangiferin treatment. These results suggested that mangiferin exerts a renoprotective effect via increasing antioxidant enzymes and reducing lipid peroxidation, which were consistent with the previous report [18].

Chronic inflammation plays an important role in the progression of DN [19]. The inflammatory cytokines, such as TNF- $\alpha$ and IL-6, are activated by the oxidative stress, PKC pathways, and chronic unresolved inflammation [31]. Previous studies have indicated that the levels of TNF- $\alpha$ and IL-6 are significantly elevated in patients with DN, as 
compared with hyperglycemic patients without nephropathy $[32,33]$. Therefore, the anti-inflammatory drugs may serve as a supplemental strategy for the treatment of renal dysfunction in diabetic patients [34]. Mangiferin has also been shown to exert a antihypoglycemic effect by modulating glucose metabolism, ameliorating insulin resistance, lowering cholesterol synthesis, and inhibiting the expression of the TNF- $\alpha$. In the present study, STZ markedly increased TNF- $\alpha$, IL$1 \beta$, and IL- 6 levels in diabetic mice, whereas the inflammatory cytokines decreased in a concentration-dependent mode by the intervention of mangiferin. Therefore, apart from antioxidative stress, the anti-inflammatory effect mediated by mangiferin is another important mechanism to protect mice from the development of DN. The outstanding effect of mangiferin on attenuating diabetic renal injury is consistent with the opinion that the anti-inflammatory and antioxidant property of mangiferin may be responsible for the alleviation of renal injuries in diabetic rats [13].

The expressions of $\alpha$-SMA protein and collagen I and IV are increased in the fibrotic kidneys of diabetic mice [8]. Fibronectin is one of the major noncollagenous glycoproteins in the extracellular matrix and basement membrane; it plays a central role in cell adhesion, regulating cell polarity, differentiation, and growth. Previous reports have indicated that mangiferin improved renal fibrosis of diabetic animals by inhibiting glomerular ECM expansion and accumulation and decreasing glomerular basement membrane thickness and mesangial cell proliferation $[18,29]$. Immunofluorescence analysis was conducted in further study to detect the change of Col I, FN, and $\alpha$-SMA. In the results, renal fibrosis characterized by significantly elevated expression levels of Col I, FN, and $\alpha$-SMA was detected in the model group, whereas mangiferin treatment significantly reversed the elevated protein expressions of Col I, FN, and $\alpha$-SMA in the diabetic mice, indicating a remarkable effect of mangiferin on renal fibrosis of DN mice. A similar study also revealed that chronic mangiferin treatment exhibited good effectiveness on renal fibrosis through decreasing Col IV and $\alpha$-SMA levels [13]. Moreover, the potential mechanisms of mangiferin effect on renal fibrosis of diabetic mice were further investigated.

TGF- $\beta$ plays a critical role in glomerulosclerosis and interstitial fibrosis; it contributes to tissue fibrosis via stimulating ECM synthesis and reducing collagenase production $[35,36]$. A variety of studies have demonstrated that TGF$\beta$ plays an important role in the pathogenesis of $\mathrm{DN}$, which is associated with oxidative stress and inflammation [3, 35, 37]. The results have shown that the intervention of mangiferin can downregulate the protein expression of TGF- $\beta 1$, indicating that mangiferin may inhibit TGF- $\beta 1$ signal activation. TGF- $\beta 1$ can enhance the deposition of ECM to initiate and promote the development of renal fibrosis [38, 39]. Therefore, this result implied that mangiferin may decrease Col I, FN, and $\alpha$-SMA by intervening the activation of TGF- $\beta 1$ relevant pathways, thereby alleviating the renal interstitial fibrosis in DN caused by STZ. These results are consistent with the previous research [40]. Similarly, the Ayurvedic antidiabetic medicine Salacia oblonga (SO) root (the major component is mangiferin) treatment reversed the increase in renal TGF- $\beta 1$ expression in the Zucker diabetic fatty (ZDF) rat kidney [35].

Phosphoinositide 3-kinase- (PI3K-) Akt signaling plays a vital role in the regulation of cell growth, metabolism, proliferation, glucose homeostasis, and vesicle trafficking [41]. One of the key mechanisms observed in the tissues impacted by type 2 diabetes is that PI3K/Akt-mediated NF- $\kappa \mathrm{B}$ signaling might be a mechanism for the treatment of DN [16]. The status of renal cortical PI3K/Akt signaling pathways was activated and higher in diabetic mice in the early phase of diabetic nephropathy [42]. Of note, other than stimulating TGF- $\beta 1$ secretion, there is also evidence indicating that IL- $1 \beta$ can activate PI3K/Akt signaling [43], thereby enhancing renal fibrosis [41]. A previous study proposed a role for $\mathrm{PI} 3 \mathrm{~K} / \mathrm{Akt}$ as a possible regulator of cell survival after mangiferin exposure [44]. Therefore, due to the potential DN related pathways, the mechanism of the PI3K/Akt signaling pathway in mangiferin inhibiting renal interstitial fibrosis was further explored. In this study, STZ prominently increased the expressions of phosphorylated PI3K and Akt, which are consistent with the conclusions reported in previous reports $[16,42]$. In addition, the administration of mangiferin significantly decreased the expression of phosphorylated PI3K and Akt in the fibrotic kidneys. A similar report also revealed that TB-II, a main ingredient of A. asphodeloides Bunge, notably improved cell viability by decreasing p-PI3K and p-Akt [28]. These findings supported that PI3K/Akt signaling may be involved in renal fibrosis induced by STZ and intervened by mangiferin.

Furthermore, a changing situation of PTEN was observed in the injured kidneys of diabetic mice. The tumor suppressor PTEN was originally identified as a negative regulator of the PI3K signaling; it can inhibit the activation of Akt [45]. The current study indicated that renal fibrosis of model mice evidenced by morphological and molecules indices in kidney was related with the downregulation of PTEN protein; however, mangiferin treatment effectively reversed these alterations. Moreover, due to the critical role of PTEN in DN, with the use of PTEN inhibitor BpV, the development of renal interstitial fibrosis was aggravated in mice models through the increased Col I, FN, $\alpha$-SMA, and TGF- $\beta 1$, as well as the elevated PI3K/AKT signaling pathway. Furthermore, the beneficial effect of mangiferin on renal interstitial fibrosis was relieved when $\mathrm{BpV}$ was presented, but not greater than without $\mathrm{BpV}$. The loss or impairment of PTEN results in an antidiabetic impact, which led to the suggestion that PTEN could be an important target for drugs against type II diabetes. In this study, when the PTEN inhibitor was used, the protein expressions of TGF- $\beta 1$, p-PI3K, and p-AKT were higher. But the expressions of PTEN change little without significance, which means the mangiferin exhibits an antifibrosis effect through regulating the activation of PTEN. The PTEN inhibitor blocks the activating effect of mangiferin. These findings demonstrated that mangiferin prevented the progression of STZ-induced DN pathology, and upregulation of PTEN may be one of the important reasons. Many animal studies and further clinical data have revealed that reduced PI3K activity can play a role in insulin sensitivity and type II diabetes [45]. Thus, one of the underlying mechanisms 
by which mangiferin attenuates DN may be to increase the insulin sensitivity by inhibiting the PI3K/Akt signaling pathway. These findings indicated that the PI3K/Akt signaling pathway exhibited a critical role in renal interstitial fibrosis, and alternative approaches which inhibited the PI3K/Akt signaling pathway and activated PTEN dependent activity might be a potential method of protecting against renal injury.

\section{Conclusions}

This study indicated that mangiferin, as an effective antifibrogenic agent, regulated the PTEN/PI3K/Akt pathway, thereby inhibiting the renal interstitial fibrosis in DN by reducing the TGF- $\beta 1$-induced elevation of $\mathrm{Col} \mathrm{I}, \mathrm{FN}$, and $\alpha$-SMA. Our findings yield novel insights into the molecular mechanisms of mangiferin in renal interstitial fibrosis and provide new therapeutic approaches for chronic fibrotic kidney disease. Mangiferin might be used as a potential adjuvant for preventing and treating DN. However, this study has some limitations. Although the expression patterns of several proteins were shown, they were not the complete molecular mechanisms involved in DN; more factors need further clarity.

\section{Data Availability}

The data used to support the findings of this study "Mangiferin Alleviates Renal Interstitial Fibrosis in StreptozotocinInduced Diabetic Mice through Regulating the PTEN/PI3K/Akt Signaling Pathway" are included within the article and available from the corresponding author upon request.

\section{Conflicts of Interest}

The authors declare that they have no conflicts of interest.

\section{Acknowledgments}

This work was supported by the National Natural Science Foundation of China (grant number 81802077) and the Science and Technology Planning Project of Jilin Province (grant number 20190701011GH).

\section{References}

[1] F. Hajiaghaalipour, M. Khalilpourfarshbafi, and A. Arya, "Modulation of glucose transporter protein by dietary flavonoids in type 2 diabetes mellitus," International Journal of Biological Sciences, vol. 11, no. 5, pp. 508-524, 2015.

[2] A. M. Hendrick, M. V. Gibson, and A. Kulshreshtha, "Diabetic retinopathy," Primary Care, vol. 42, no. 3, pp. 451-464, 2015.

[3] S. Zhang, H. Xu, X. Yu, Y. Wu, and D. Sui, "Metformin ameliorates diabetic nephropathy in a rat model of low-dose streptozotocin-induced diabetes," Experimental and Therapeutic Medicine, vol. 14, no. 1, pp. 383-390, 2017.

[4] I. I. Witzel, H. Jelinek, K. Khalaf, S. Lee, A. H. Khandoker, and H. Alsafar, "Identifying common genetic risk factors of diabetic neuropathies," Frontiers in Endocrinology, vol. 6, p. 88, 2015.
[5] A. Nogueira, M. J. Pires, and P. A. Oliveira, "Pathophysiological mechanisms of renal fibrosis: a review of animal models and therapeutic strategies," In Vivo, vol. 31, no. 1, pp. 1-22, 2017.

[6] O. M. Akchurin and F. Kaskel, "Update on inflammation in chronic kidney disease," Blood Purification, vol. 39, no. 1-3, pp. 84-92, 2015.

[7] P. B. Pal, K. Sinha, and P. C. Sil, "Mangiferin attenuates diabetic nephropathy by inhibiting oxidative stress mediated signaling cascade, TNF $\alpha$ related and mitochondrial dependent apoptotic pathways in streptozotocin-induced diabetic rats," PLoS One, vol. 9, no. 9, article e107220, 2014.

[8] Y. Wang, L. Jia, Z. Hu, M. L. Entman, W. E. Mitch, and Y. Wang, "AMP-activated protein kinase/myocardin-related transcription factor-A signaling regulates fibroblast activation and renal fibrosis," Kidney International, vol. 93, no. 1, pp. 81-94, 2018.

[9] C. Ho, P. H. Lee, Y. C. Hsu, F. S. Wang, Y. T. Huang, and C. L. Lin, "Sustained Wnt/ $\beta$-catenin signaling rescues high glucose induction of transforming growth factor- $\beta 1$-mediated renal fibrosis," The American Journal of the Medical Sciences, vol. 344, no. 5, pp. 374-382, 2012.

[10] K. R. Tuttle, "Linking metabolism and immunology: diabetic nephropathy is an inflammatory disease," Journal of the American Society of Nephrology, vol. 16, no. 6, pp. 1537-1538, 2005.

[11] L. C. Cantley, "The phosphoinositide 3-kinase pathway," Science, vol. 296, no. 5573, pp. 1655-1657, 2002.

[12] J. Zhu, N. Sun, L. Aoudjit et al., "Nephrin mediates actin reorganization via phosphoinositide 3-kinase in podocytes," Kidney International, vol. 73, no. 5, pp. 556-566, 2008.

[13] X. Zhu, Y. Q. Cheng, L. du et al., "Mangiferin attenuates renal fibrosis through down-regulation of osteopontin in diabetic rats," Phytotherapy Research, vol. 29, no. 2, pp. 295-302, 2015.

[14] M. Imran, M. S. Arshad, M. S. Butt, J. H. Kwon, M. U. Arshad, and M. T. Sultan, "Mangiferin: a natural miracle bioactive compound against lifestyle related disorders," Lipids in Health and Disease, vol. 16, no. 1, p. 84, 2017.

[15] A. Dar, S. Faizi, S. Naqvi et al., "Analgesic and antioxidant activity of mangiferin and its derivatives: the structure activity relationship," Biological \& Pharmaceutical Bulletin, vol. 28, no. 4, pp. 596-600, 2005.

[16] J. N. Hong, W. W. Li, L. L. Wang et al., "Jiangtang decoction ameliorate diabetic nephropathy through the regulation of $\mathrm{PI} 3 \mathrm{~K} /$ Akt-mediated NF- $\kappa \mathrm{B}$ pathways in KK-Ay mice," Chinese Medicine, vol. 12, no. 1, p. 13, 2017.

[17] S. Muruganandan, S. Gupta, M. Kataria, J. Lal, and P. K. Gupta, "Mangiferin protects the streptozotocin-induced oxidative damage to cardiac and renal tissues in rats," Toxicology, vol. 176, no. 3, pp. 165-173, 2002.

[18] Y. W. Liu, X. Zhu, L. Zhang et al., "Up-regulation of glyoxalase 1 by mangiferin prevents diabetic nephropathy progression in streptozotocin-induced diabetic rats," European Journal of Pharmacology, vol. 721, no. 1-3, pp. 355-364, 2013.

[19] C. Mora and J. Navarro, "Inflammation and diabetic nephropathy," Current Diabetes Reports, vol. 6, no. 6, pp. 463-468, 2006.

[20] T. Szkuldeshi, "The mechanism of alloxan and streptozotocin action in B cells of the rat pancreas," Physiological Research, vol. 50, pp. 537-546, 2001. 
[21] S. Lenzen, "The mechanisms of alloxan- and streptozotocininduced diabetes," Diabetologia, vol. 51, no. 2, pp. 216-226, 2008.

[22] F. Dupuis, J. Atkinson, P. Limiñana, and J. M. Chillon, "Captopril improves cerebrovascular structure and function in old hypertensive rats," British Journal of Pharmacology, vol. 144, no. 3, pp. 349-356, 2005.

[23] K. L. Wang, "Research progress of the relationship between serum uric acid level and diabetic nephropathy in patients with type 2 diabetes mellitus," Hainan Medical Journal, vol. 24, no. 4, pp. 51-59, 2013.

[24] G. L. Chen, W. Wei, and S. Y. Xu, "Effect and mechanism of total saponin of Dioscorea on animal experimental hyperuricemia," The American Journal of Chinese Medicine, vol. 34, no. 1, pp. 77-85, 2006.

[25] M. Herman-Edelstein, P. Scherzer, A. Tobar, M. Levi, and U. Gafter, "Altered renal lipid metabolism and renal lipid accumulation in human diabetic nephropathy," Journal of Lipid Research, vol. 55, no. 3, pp. 561-572, 2014.

[26] T. Chen, J. Gao, P. Xiang et al., "Protective effect of platycodin $\mathrm{D}$ on liver injury in alloxan-induced diabetic mice via regulation of Treg/Th17 balance," International Immunopharmacology, vol. 26, no. 2, pp. 338-348, 2015.

[27] F. Li, Y. L. Yuan, C. R. Guo et al., "Timosaponin B-II ameliorates diabetic nephropathy via TXNI $\mathrm{P}, \mathrm{mTOR}$, and NF- $\kappa \mathrm{B}$ signaling pathways in alloxan-induced mice," Drug Design, Development and Therapy, vol. 9, pp. 6247-6258, 2015.

[28] Y.-L. Yuan, B. Q. Lin, C. F. Zhang et al., "Timosaponin B-II ameliorates Palmitate-Induced insulin resistance and inflammation via IRS-1/PI3K/Akt and IKK/NF- $\kappa \mathrm{B}$ pathways," The American Journal of Chinese Medicine, vol. 44, no. 4, pp. 755-769, 2016.

[29] X. Li, X. Cui, X. Sun, X. Li, Q. Zhu, and W. Li, "Mangiferin prevents diabetic nephropathy progression in streptozotocininduced diabetic rats," Phytotherapy Research, vol. 24, no. 6, pp. 893-899, 2010.

[30] M. Brownlee, "The pathobiology of diabetic complications: a unifying mechanism," Diabetes, vol. 54, no. 6, pp. 16151625, 2005.

[31] M. Brownlee, "Biochemistry and molecular cell biology of diabetic complications," Nature, vol. 414, no. 6865, pp. 813-820, 2001.

[32] K. Sekizuka, Y. Tomino, C. Sei et al., "Detection of serum IL-6 in patients with diabetic nephropathy," Nephron, vol. 68, no. 2, pp. 284-285, 1994.

[33] J. Navarro et al., "The role of TNF- $\alpha$ in diabetic nephropathy: Pathogenic and therapeutic implications," Cytokine \& Growth Factor Reviews, vol. 17, no. 6, pp. 441-450, 2006.

[34] A. Mima, "Inflammation and oxidative stress in diabetic nephropathy: new insights on its inhibition as new therapeutic targets," Journal of Diabetes Research, vol. 2013, Article ID 248563, 8 pages, 2013.

[35] L. He, Y. Qi, X. Rong et al., “The Ayurvedic Medicine Salacia oblonga Attenuates Diabetic Renal Fibrosis in Rats: Suppression of Angiotensin II/AT1 Signaling," Evidence-Based Complementary and Alternative Medicine, vol. 2011, Article ID 807451, 12 pages, 2011.

[36] G. Wolf, "Renal injury due to renin-angiotensin-aldosterone system activation of the transforming growth factor-beta pathway," Kidney International, vol. 70, no. 11, pp. 1914-1919, 2006.
[37] H. B. Lee, M. R. Yu, Y. Yang, Z. Jiang, and H. Ha, "Reactive oxygen species-regulated signaling pathways in diabetic nephropathy," Journal of the American Society of Nephrology, vol. 14, 8, Supplement 3, pp. S241-S245, 2003.

[38] H. W. Schnaper, T. Hayashida, S. C. Hubchak, and A. C. Poncelet, "TGF- $\beta$ signal transduction and mesangial cell fibrogenesis," American Journal of Physiology-Renal Physiology, vol. 284, no. 2, pp. F243-F252, 2003.

[39] A. Bürger, C. Wagner, C. Viedt, B. Reis, F. Hug, and G. M. Hänsch, "Fibronectin synthesis by human tubular epithelial cells in culture: effects of PDGF and TGF- $\beta$ on synthesis and splicing," Kidney International, vol. 54, no. 2, pp. 407-415, 1998.

[40] A. C. Han, J. X. Deng, Q. S. Huang et al., "Verapamil inhibits scar formation after peripheral nerve repair in vivo," Neural Regeneration Research, vol. 11, no. 3, pp. 508-511, 2016.

[41] H. E. Yoon, S. J. Kim, S. J. Kim, S. Chung, and S. J. Shin, “Tempol attenuates renal fibrosis in mice with unilateral ureteral obstruction: the role of PI3K-Akt-FoxO3a signaling," Journal of Korean Medical Science, vol. 29, no. 2, pp. 230-237, 2014.

[42] D. Feliers, S. Duraisamy, J. L. Faulkner et al., "Activation of renal signaling pathways in $\mathrm{db} / \mathrm{db}$ mice with type 2 diabetes," Kidney International, vol. 60, no. 2, pp. 495-504, 2001.

[43] A. Donaldson, D. Daphna-Iken, T. Tetsuka, and A. R. Morrison, "Interleukin-1 beta activates PI 3-kinase in renal mesangial cells," Biochemical and Biophysical Research Communications, vol. 227, no. 1, pp. 289-293, 1996.

[44] S. Saha, P. Sadhukhan, K. Sinha, N. Agarwal, and P. C. Sil, "Mangiferin attenuates oxidative stress induced renal cell damage through activation of PI3K induced Akt and Nrf-2 mediated signaling pathways," Biochemistry and Biophysics Reports, vol. 5, pp. 313-327, 2016.

[45] A. Carracedo and P. P. Pandolfi, "The PTEN-PI3K pathway: of feedbacks and cross-talks," Oncogene, vol. 27, no. 41, pp. 5527$5541,2008$. 